\title{
Hole drilling and milling of magnetic alloys parts by shaped tube electrolytic machining
}

\author{
Vladimir V. Glebov*, Irina N. Danilenko, and Ruslan I. Ratushinsky \\ Don State Technical University, 344000 Rostov-on-Don Gagarin sq. 1, Russia
}

\begin{abstract}
In this research shaped tube electrolytic machining of drilling and milling of magnetic alloys parts and difficult-to-cut metals, steels and alloys is presented. New research made in the field of space, aviation, automobile, medical, computer and electronics, and others has created the need for small and fine holes with high aspect ratio in these materials. The primary investigations of ECM with the tubular tool electrode are presented. Compared with mechanical machining, shaped tube electrolytic machining (STEM) exhibits an advantage in producing micro-holes with a high aspect ratio and in producing the curved holes. In order to realize the process of electrochemical machining, experimental assembly with the shaped tube tool electrode has been designed and manufactured. Completed researches indicate that this tool electrode has a high potential to machine difficult-to-cut and brittle metals economically and efficiently.
\end{abstract}

\section{Introduction}

Modern industry uses a large range of hard-to-process materials, as well as special surface coatings of parts that have high heat resistance, increased strength, toughness, corrosion resistance, high magnetic properties, etc. For many parts of such materials, conventional machining methods, such as milling or grinding, are not applicable or effective. For these purposes, non-traditional methods of machining, such as laser, ultrasonic, electrodischarge, electrochemical, etc., are increasingly used. Electrochemical machining (ECM) is widely used in machine building, especially in aircraft engine building, defense industries, machine tool building, etc. ECM methods can handle any metals and alloys, the process does not depend on the mechanical properties of the material being processed, its hardness, viscosity, brittleness [1-4].

Nevertheless, ECM methods are not widely universal. The equipment for ECM of various parts makes up $1.5 \%$ of the entire fleet of machine tools in machine building. This is due to the increased energy intensity $(10-30 \mathrm{kWh} / \mathrm{kg})$ and the complexity of the process, the relatively high cost of equipment and rigging, a significant time to prepare the production [2-3]. However, in the workshops for processing blades of turbines and compressors of aircraft engine plants, the share of ECM machines reaches $15-20 \%$.

\footnotetext{
* Corresponding author: glebovdon@mail.ru
} 
ECM is based on the principle of anodic etching of metals in an electrolyte medium. The workpiece is an anode, and a positive supply pole is fed to the tool electrode (TE). The process takes place in the inter-electrode gap (IEG) at short distances of the order of $0.1 \mathrm{~mm}$ in the electrolyte medium.

Deep drilling, milling and thin cutting of materials presents a serious problem in modern production, especially for traditional mechanical processing methods. Various nozzles used in aviation and space technology are made of wear-resistant heat-resistant and corrosion-resistant materials, and the flow-through part of these products must be made with high accuracy and purity of processing for working with corrosive media and under stringent conditions of thermodynamic and vibrational loads [3, 5-7].

In the present work, the possibility of electrochemical drilling and obtaining and processing internal channels of difficult-to-cut metals, steels and alloys was investigated. The high hardness of blanks from high-strength and magnetic alloys with small diameters of internal holes does not allow them to be formed by the traditional method - drilling with subsequent grinding.

\section{Theoretical analysis}

Hole drilling and cutting of difficult-to-cut metals, semiconductor and magnetic alloys parts can be performed with the help of wire TE during the supply of electrolyte along the IEG $[2,3,10]$. On the part of the part, where the jet of electrolyte is directed, the metal is etched with the formation of depressions up to 1-1.5 $\mathrm{mm}$. Due to various perturbations, inhomogeneities, etc. there is turbulence of the motion of the jet. Such a method does not allow cutting slabs with a thickness of more than $30 \mathrm{~mm}$ due to disturbance of the hydrodynamic regime along the length of each part of the wire. In this case, the width of the cut at the inlet of the jet is less than at the outlet. The top edges of the cut are sharp, the lower edges are rounded, the radius of rounding is $0.2-0.5 \mathrm{~mm}$. The cut has a taper caused by a number of reasons: the jet cone, the heating of the electrolyte in the gap, the additional expansion of the jet due to its friction against the cutting wall, the release of gas, etc. When cutting workpieces with a height of $11 \mathrm{~mm}$, the machining accuracy was $\pm 0.07 \mathrm{~mm}$, the taper angle of the cut was $2^{\circ}$, and the surface roughness was $1-2 \mu \mathrm{m}[2,5]$.

At present, a number of another methods are known [3, 5, 6, 8-10], with the help of which electrochemical drilling, milling and cutting of metals can be carried out. The accuracy of processing in the electrochemical cutting method is mainly determined by the stability of the geometric parameters of the jet along the height of the cut and in time. In this regard, when developing the process of electrochemical cutting, it is first of all necessary to solve the problem of the possibility of obtaining a stable jet moving with large kinetic energy.

In the known methods $[3,8]$, cutting and cutting of metals is carried out by a tube TE having longitudinal narrow slots through which an electrolyte is supplied perpendicular to the treatment front, i.e. in the direction of the cut. Obviously, this method is technologically difficult to implement. A high pressure of the electrolyte is also required, and because of the pressure drop along the tube axis and the difference in the electrolyte distribution along the height of the billet, the quality of the surface to be treated when cutting large-thickness parts is reduced, and the cut turns out to be sufficiently wide.

To improve the technological parameters of the cut, a free flow of the jet through the gap is necessary, without a hydraulic shock. The use of disk TE [3] when cutting parts does not eliminate these drawbacks. The deterioration of the hydrodynamic regime when cutting large thickness parts leads to frequent closures. The width of the cut exceeds the thickness of the disc by $0.25-0.40 \mathrm{~mm}$. Therefore, a combined method of mechanical and electrochemical cutting using diamond disks on a metal base is often used $[3,6,10]$. 


\section{Experimental part}

The carried out researches have shown that to expand the possibility of electrochemical cutting of metals along the height of the billet, to improve the quality of surface treatment, it is advisable to use a tubular TE having a groove in the lateral surface with the supply of electrolyte along the IEG. Experimental studies of the cutting process showed that the best quality at the maximum cutting height is obtained by using a tube TE having a groove, the height of the cut part of which varies along the inner radius of the tube within $0.5 R \leq h \leq R$ (Fig. 1). The height of the IEG was determined by the height of the part of the current electrolyte protruding from the TE.

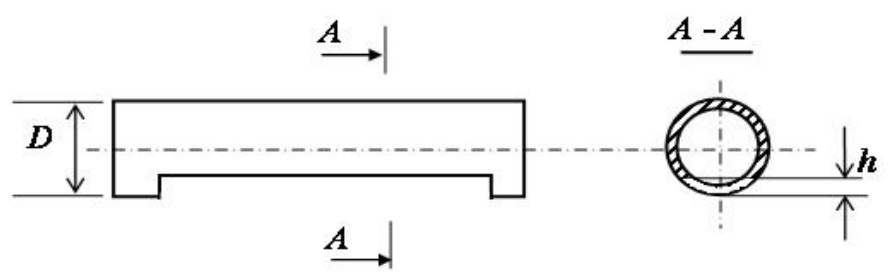

Fig. 1. Tubular TE for cutting and milling of metal products

The proposed form of tubular TE contributes to the formation of the necessary configuration of the electrolyte stream when it is fed along the IEG and maintains the required hydrodynamic regime along the entire length of the treated surface [2-5, 11-12]. The accuracy of the cut was determined with the help of the MIR-1M reference microscope, the surface roughness was determined by the standard method in the same way as in the case of hole piercing. Samples were weighed before and after the experiment on a laboratory scale VLDP-100 g with a $0.05 \mathrm{mg}$ fission rate for determining the current yield. The height of microroughness and the depth of removal were measured with a profilographprofilometer M-201, the quality and shape of the treated surface were studied with the help of the microscope MMU-3U-4.2.

The technological capabilities of the process were tested by processing internal channels, drilling and cutting workpieces in the form of a rectangular prism $50 \mathrm{~mm}$ high and a hollow cylindrical billet $55 \mathrm{~mm}$ high with an outer diameter of $30 \mathrm{~mm}$ and an internal cylindrical billet of magnetic alloys of YuNDK24, YuND4, FeCoCr. In view of the extremely high brittleness, hardness and the presence of considerable internal stresses, mechanical grinding leads to the appearance on the surface being treated of chips, cracks, to the discoloration of individual grains and metal particles, to considerable wear of grinding wheels and other negative consequences. This is the reason for the production waste products [13-15].

At the initial stage of the study, the anode behavior of cast alloys was studied for the choice of electrolyte from the dependence of the current density on the anode potential (polarization curves). The measurements were carried out on a rotating disc electrode $(\mathrm{n}=2200 \mathrm{rpm})$, at a $\mathrm{P} 5484 \mathrm{M}$ potentiostat with a scan rate of $2.4 \mathrm{~V} / \mathrm{min}$ in the range from 0 to $4 \mathrm{~V}$, with the registration of the potentiodynamic curves on a two-coordinate plotter. A saturated silver chloride electrode $(\varphi=0.222 \mathrm{~V})$ was used as the reference electrode. To obtain a low surface roughness of the alloys, a localizing electrolyte, a $10 \%$ solution of sodium nitrate, was selected for which the anode-anion activation of the alloys began at an increased potential near $1.8 \mathrm{~V}$ (for comparison, $0.7 \mathrm{~V}$ for an aqueous solution of $15 \% \mathrm{NaCl}$, the process takes place in the active region). The $\mathrm{pH}$ value was determined using a PLU-01 $p H$ meter with $\mathrm{R}_{\text {in }}$ of at least $10^{11} \mathrm{Ohm}$. 
Cutting was carried out by a thin-walled cylindrical tube (wall thickness $0.2 \mathrm{~mm}$ ) with an internal diameter of $0.6 \mathrm{~mm}$. The lateral groove was obtained by removing a part of the surface with $\mathrm{h}=0.6 \mathrm{R}=0.18 \mathrm{~mm}$. The pressure at the entrance to the IEG was $5 \times 10^{5} \mathrm{~Pa}$, the processing current for cutting the prismatic workpiece was $30 \mathrm{~A}$, and for cutting the hollow cylinder it varied from 0 to $25 \mathrm{~A}$. The cutting speed was $0.8 \mathrm{~mm} / \mathrm{min}$. The typical width of the cut was $2.00 \pm 0.05 \mathrm{~mm}$. Cutting edges along the entire height of the workpiece were sharp.

The experimental setup designed and developed as shown in Figure 2 a) was used for hole drilling, milling and cutting of difficult-to-cut metals, semiconductor billets and magnetic alloys parts. The process of ECM occurred directly in the electrolytic bath, which is a prismatic body with overall dimensions of $290 \times 290 \times 210 \mathrm{~mm}$, made of $32 \mathrm{~mm}$ thick acrylic plastic.

To carry out research on the drilling of parts, various tubular TI, shown in Figure 2 b), were used. For example, the outer diameter of the TI was $5.40 \mathrm{~mm}$, the diameter of the hole for supplying the electrolyte was $2.5 \mathrm{~mm}$.

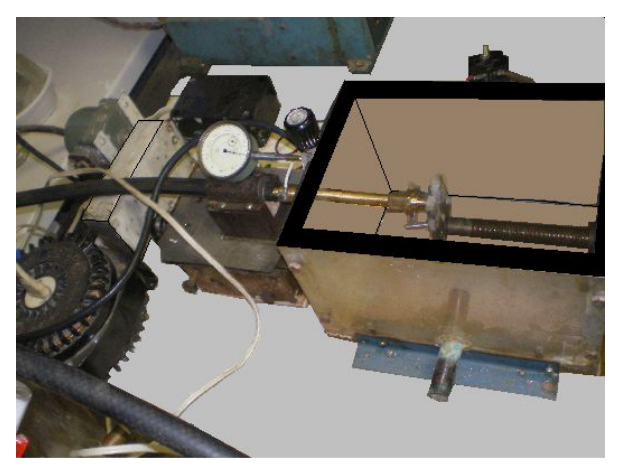

a)

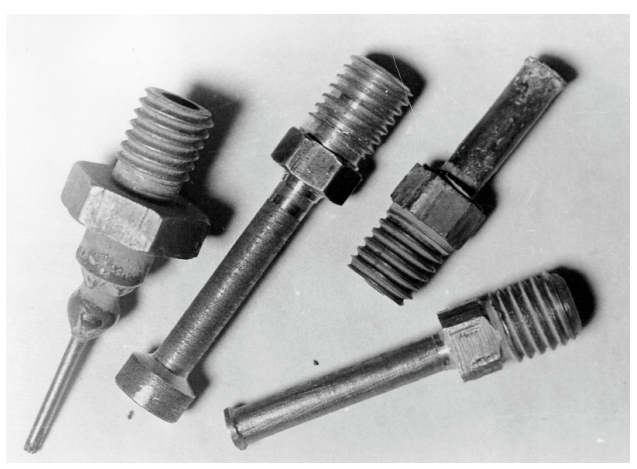

b)

Fig. 2. a) Equipment for ECM; b) TE for hole drilling

To improve the insulation of the lateral surface, a polyvinylchloride tube was applied over the entire length of the TE. When working with such TE, it was found out that this insulation serves not only as a dielectric, but also creates a kind of counterpressure chamber. The quality of the treated surface when working with such TE has improved dramatically. An important role in the accuracy of the treatment is played by the correct choice of the IEG value. If the IEG is more than optimal, then a strong erosion of the input is obtained, and if the IEG is too small, there is a strong sparking. The structural inhomogeneities and microroughness on the outer surface of the samples are affected. The selected IEG was about $0.2 \mathrm{~mm}$, for comparative analyzes it did not change during all the studies.

Taper angle decreased, because the lateral part of the TE received good insulation and the erosion of the lateral part became completely insignificant. The strength of such a coating is much higher than previously used. Even after 5-6 repetitions of the process, the insulation was not significantly disturbed. The firmware of such TE was successfully carried out both on fine-grained samples (FeCoCr, Alnico (YuNDK24)), and on coarsegrained samples (such as Alni (YuND4)). The accuracy of hole drilling also increased, for example, if the diameter of the TE was $6.3 \mathrm{~mm}$, the input diameter of the resulting hole was $7.0 \mathrm{~mm}$ and the gap was about $0.35 \mathrm{~mm}$. After drilling, small annular thickenings are 
sometimes observed inside the hole along the surface, the appearance of which is probably due to a change in the pressure of the electrolyte in the system.

\section{Conclusion}

Thus, in this work, an experimental design of a tubular electrode tool is proposed for electrochemical piercing and cutting of hard-to-digestible materials. The main advantages of the proposed method of cutting in comparison with the method of cutting wire TE:

- The more important parameters that influence hole size and taper angle in shaped tube electrolytic machining are electrolyte pressure, feed speed TE, current density, and composition and concentration of electrolyte;

- Expand the possibility of cutting workpieces in height;

- Improving the quality of the treated surface, achieved by stabilizing the hydrodynamic regime.

To improve the quality of the holes to be pierced, to stabilize the processing parameters, to reduce the temperature drop and the electrical conductivity along the IEG, it is necessary to achieve a constant rate of flow of the electrolyte and excess pressure in the IEG, which varies as the TE moves in the billet. Technically, this can be done by creating a backpressure of the electrolyte in the ECM chamber.

The accuracy and quality of ECM depend significantly not only on the chemical composition of the alloy, but also on the physical and chemical structure of the material being processed. Therefore, for practical use, preliminary optimization of technological, electrochemical and hydrodynamic parameters of the process for each material is necessary.

\section{References}

1. V.V. Glebov, Procedia Engineering, 129, 510 (2015)

2. M. Sen, H.S. Shan, Int. J. Mach. Tools Manuf., 45 (2), 137 (2015)

3. V.V. Glebov, Procedia Engineering, 206, 918 (2017)

4. S.V. Kirsanov, V.V. Glebov, Yu.V. Prysjagnuk, Metallobrabotka (Metal Working), 20.2, 26 (2004)

5. F.I. Kukoz, V.V. Glebov, S.V. Kirsanov, V.V. Konovalenko, Russian Journal of Electrochemistry, 32 (9), 1060 (1996)

6. V.V. Glebov, Ju.V. Prisyazhnyuk, L.A. Kaplin, Nauchno-tehnicheskij Vestnik Povolzh'ja, 5, 140 (2012)

7. K.P. Rajurkar, M.M. Sundaram, A.P. Malshe, Procedia CIRP, 6, 13 (2013)

8. S.V. Kirsanov, V.V. Glebov, Elektronnaya Obrabotka Materialov, 5, 4 (2004)

9. A.Sh. Bayramyan, G.G. Gevorkyan, F.A. Harutyunyan, Elektronnaya Obrabotka Materialov, 5-6, 80 (1965)

10. I.V. Batinov, Intellectual Systems in Production, 1 (11), 244 (2008)

11. D.S. Bilgi, R. Kumar, V.K. Jain, R. Shekhar, Int. J. of Adv. Man. Techn., 39, 47 (2008)

12. Ph.I. Kukoz, S.V. Kirsanov, V.V. Glebov, Elektronnaya Obrabotka Materialov, 4, 4 (2000)

13. V.V. Glebov, Inženernyj vestnik Dona (Rus), 2 (2), URL: www.ivdon.ru/ru/magazine/archive/n2p2y2015/2966 (2015)

14. V.V. Glebov, Ju.V. Prisyazhnyuk, Sovremennye Problemy Nauki i Obrazovaniya, 4, URL: www.science-education.ru/98-4758 (2011)

15. V.V. Glebov, Yu.V. Prysjagnuk, S.V. Kirsanov, Kazanskaya Nauka, 2, 31 (2011) 